\title{
Traf: A Camera based System for Traffic Flow Analysis
}

\author{
Shibing Feng ${ }^{a}$, Jian Tang ${ }^{b}$, Ming Zhao ${ }^{c}$, Zhen Zhang ${ }^{d}$ and Hengchang Liu e \\ Department of Software Engineering, University of Science and Technology of China, Hefei, 230026, \\ China \\ afengshibing0@163.com, btangjiandna@163.com, 'zhaoming626@yeah.net, \\ dsa514015@mail.ustc.edu.cn, ehcliu1984@vip.163.com
}

\section{Keywords: Traffic Flow; Video Analysis; Background Subtraction}

\begin{abstract}
As the traffic jam is becoming a serious issue of urban life, collecting traffic parameters for traffic distribution is important and critical. In this paper, we present a camera based system (Traf) to count automotive vehicles for traffic flow analysis use. The system employs the method of background subtraction by comparing differences between foreground and background. The subtraction result is utilized to identify the position and the shape of vehicles. The background model is initialed by averaging first 50 frames, and adapted to environment change by introducing an adapting parameter $\lambda$. Compared to state-of-art method, Traf is more robust, adaptive and economical.
\end{abstract}

\section{Introduction}

With the development of economy, automobile vehicle number is increasing sharply, which brings many traffic jams and increases traffic management cost [1]. One of the most feasible ways to solve these problems is to gather the traffic parameters effectively and distribute the resources of road reasonably [2], [3]. The video image based algorithm utilizes computer vision technology [4] and cyber physical system, providing an efficient and economical solution towards this issue.

Different from most rest methods, video image is capable to provide rich information, and it is easy to deploy to varies environment. Besides, the collected information is convenient to store and share, making it possible for traffic monitor, accident analysis and so on [5]. What's more, video based method is friendly for further upgrading. While these advantages are hard to achieve by ultrasonic [6], infrared [7] or magnetic performance [8].

Currently, there are lots of cameras on the road for traffic monitor, which can hence provide rich original video sources. Lots of methods for moving object recognition and tracking in the intelligent traffic monitoring system was proposed, like frame subtraction [9], but its accuracy is questionable due to the overlapping. Our system is designed to work on the video collected by cameras on the road, and we employed background subtraction algorithm [10] which is efficient and reliable.

\section{System Overview}

The system consists of 3 main modules, video collector, vehicle counter and flow analyzer as Fig1. Video collector is designed to collect the original video sources and transform them into a unified format for further use. Flow analyzer is designed to calculate the result and provide suggestion for traffic use. The vehicle counter is the core module of the system, which is further divided into 2 steps as, detector and tracker. 

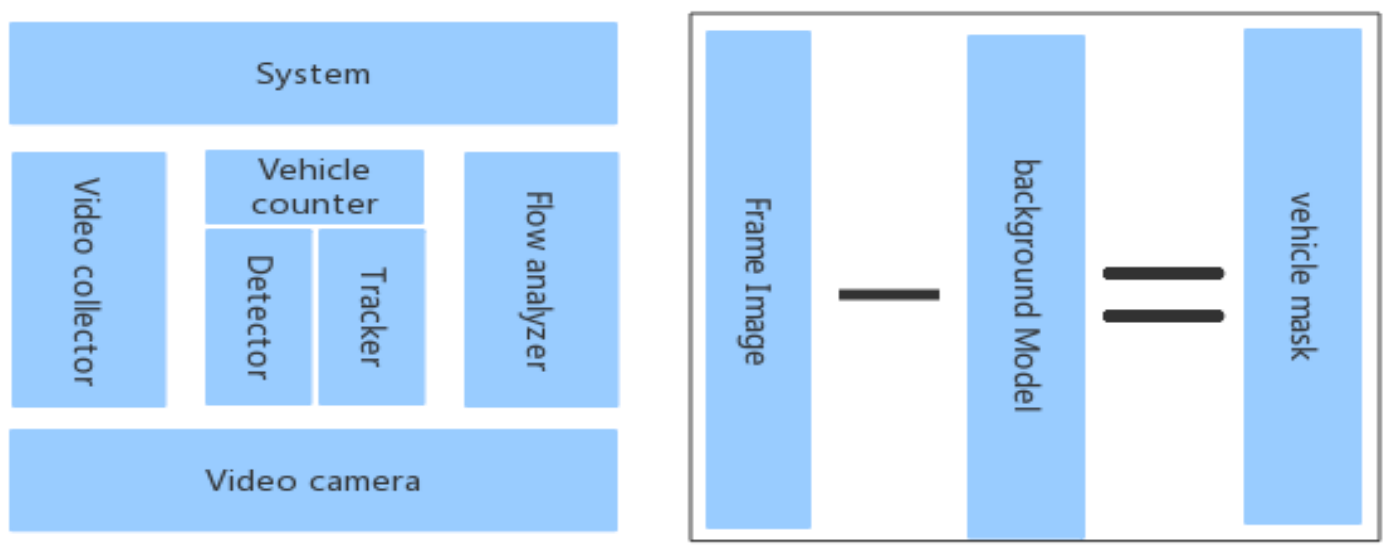

Fig.1. System diagram: Video collector - Vehicle counter - Flow analyzer

\section{Background Subtraction}

As the monitoring camera is deployed with a fixed position, videos collected from this kind of devices would have a stable background. And the automobile vehicles form the foreground motions. Hence, content of each frame image contains two parts, foreground and background. To detect the vehicles, we utilize background subtraction method, which can be described as:

$$
F=I-B
$$

Where $I$ is derived from frame image, $B$ is the corresponding background, and $F$ is hence the detected foreground.

Frame images from video usually have more than one channel, and they may vary from different types and sizes. This makes it hard or impossible for directing subtraction calculation. Hence, we introduce a collector module to unify the original frame images.

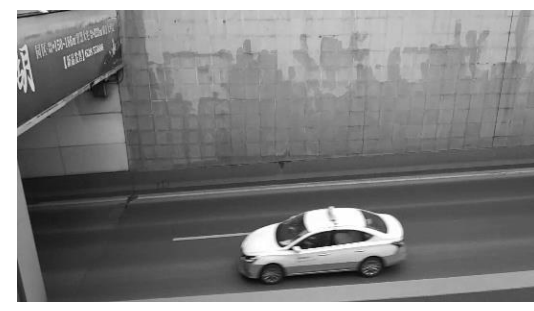

(a) Image frame

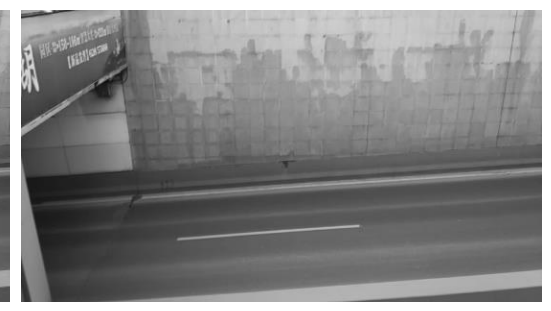

(b) Background model

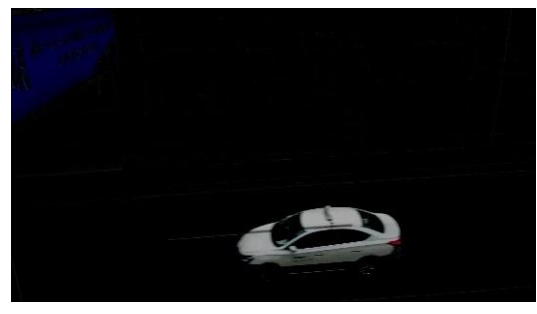

(c) Foreground image

Fig.2. Subtracting background from image frame, the result is foreground image, or vehicle mask.

After unifying operation, we do background subtraction as Fig.2. The result foreground image Fig.2(c) is mainly consisted of 2 kinds of area, the black and the gray. They stand for the background and the vehicle separately. However, the gray parts are distributed all the image, making it hard for automobile vehicle detection. Ideally, the gray area should gather together as an island. But due to camera performance, environment disturbance and so on, the image carries noise. Even worse, some parts of automobile vehicle may have coincident color with the background overlapping, this would result in gabs and separations.

$$
\text { mask }=\operatorname{sign}(v)= \begin{cases}1, & V \geq \delta \\ 0, & v<\delta\end{cases}
$$

To solve the problem above, we take following strategies as Fig.3. First, we employ a Gaussian filter with $4 * 4$ size window to reduce noise. Second, we transform the result into binary image with Eq.2, the result mask may consist of several neighbor areas. To make it a nice mask for describing automobile vehicles' shapes and positions, we employ dilation. Finally, we apply erosion for a better result. 


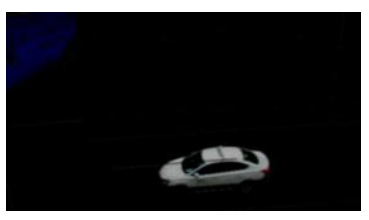

(a) Filter Gaussian noise

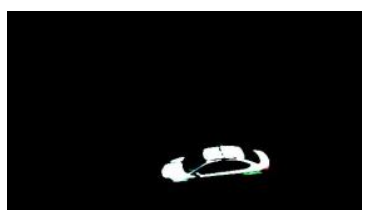

(b) Binary image

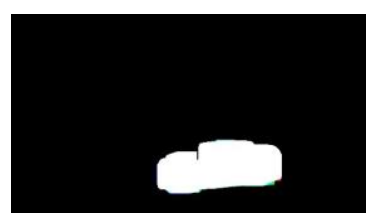

(c) Dilation

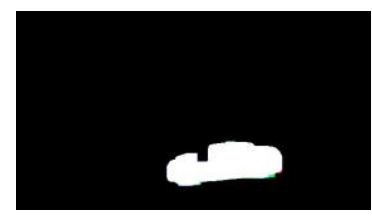

(d) Erosion

Fig.3. Gaussian Filter - Binary Image - Dilation - Erosion

After preview operations, vehicle mask becomes a binary image consists of white islands and black background. The white symbolizes vehicle cars within foreground, and we detect the motion by counting the contour of the island. Considering the size and the shape of the automobile vehicle, it's obviously that islands with small area should be expelled. Then we track the detected motion with Kalman-filter, hence we record the vehicle exactly once for appearing in the view.

\section{Background Model}

One novel point of our system is the background model. As frame images consists of two parts, foreground and background. If there were no automobile vehicles within the frame image, the background is hence equal to the frame image. But this is not always the case, as the automobile vehicles are running on the road from time to time. Even worse, background may change due to light, wind and so on. So, a steady background model is essential to the robustness of the system. Traf first builds the initial background model by averaging first 50 frame images. The result is shown as Fig.4.

To adapt to environment change, Traf employs an adaptive parameter $\lambda$ as Eq.3.

$M_{t}=(1-\lambda) M_{t-1}+\lambda B_{t}$

where $M_{t}$ and $M_{t-1}$ are the background model at time $\mathrm{t}$ and $\mathrm{t}-1, B_{\mathrm{t}}$ is the background derived from frame image at time $\mathrm{t}$.

$$
\begin{aligned}
& F_{t}=I_{t}-M_{t-1} \\
& B_{t}=y\left(F_{t}\right) * M_{t-1}+\left(1-y\left(F_{t}\right)\right) I_{t}(5)
\end{aligned}
$$

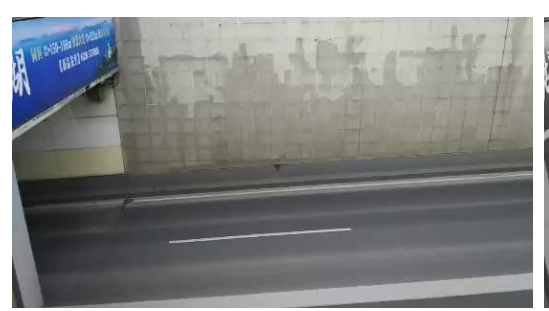

(a)Background in color

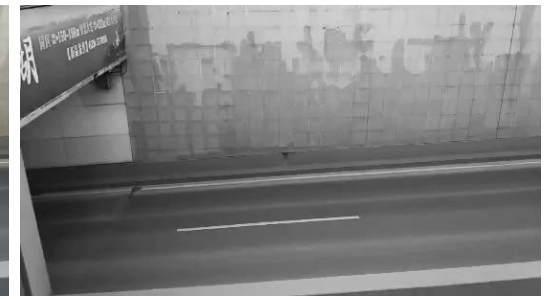

(b) Background in gray

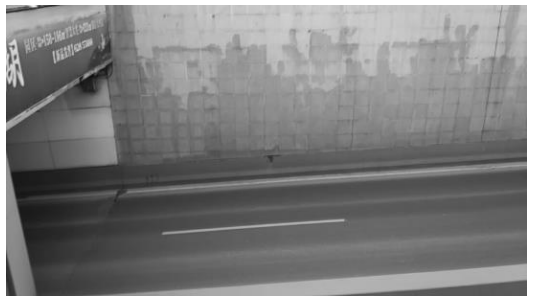

(c) Background model

Fig.4. Background constructing and updating

Item $B_{t}$ is built from the mask of foreground and background model at time t. The mask is derived from a sign function as:

$$
y=\operatorname{sign}(F)= \begin{cases}1, & F \geq \varepsilon \\ 0, & F<\varepsilon\end{cases}
$$

\section{Conclusion}

This paper presents the design, implementation and evaluation of a reliable traffic parameter collector based on camera video, which is efficient and affordable. It employs a novel background model building and updating algorithm, making the system be available for varies environment. Compare to state-of-art frame subtraction method, our system can achieve an accurate and real-time result. 


\section{Acknowledgements}

In this paper, the research was sponsored by the National Nature Science Foundation of China (Project No. 61472384).

\section{References}

[1] Yuki Sugiyama, Minoru Fukui, Macoto Kikuchi, Katsuya Hasebe, Akihiro Nakayama, Katsuhiro Nishinari, Shin-ichi Tadaki, and Satoshi Yukawa. Traffic jams without bottlenecks experimental evidence for the physical mechanism of the formation of a jam. New journal of physics, 10(3):033001, 2008.

[2] Bingbing Ni, Gang Wang, and Pierre Moulin. Rgbd-hudaact: A color-depth video database for human daily activity recognition. In Consumer Depth Cameras for Computer Vision, pages 193-208. Springer, 2013.

[3] Stelios Timotheou, Christos G Panayiotou, and Marios M Polycarpou. Distributed traffic signal control using the cell transmission model via the alternating direction method of multipliers. IEEE Transactions on Intelligent Transportation Systems, 16(2):919-933, 2015.

[4] Jun Yang, Man-Woo Park, Patricio A Vela, and Mani Golparvar-Fard. Construction performance monitoring via still images, time-lapse photos, and video streams: Now, tomorrow, and the future. Advanced Engineering Informatics, 29(2):211-224, 2015.

[5] Heather N Zaccaro, Emily C Carbone, Nishita Dsouza, Michelle R Xu, Mary C Byrne, and John D Kraemer. Assessing the reliability and validity of direct observation and traffic camera streams to measure helmet and motorcycle use. Injury prevention, 21(6):415-417, 2015.

[6] Youngtae Jo, Jinsup Choi, and Inbum Jung. Traffic information acquisition system with ultrasonic sensors in wireless sensor networks. International Journal of Distributed Sensor Networks, 10(5):961073, 2014.

[7] Joshua Harrison, Kurtulus, 'Izzetog`lu, Hasan Ayaz, Ben Willems, Sehchang Hah, Ulf Ahlstrom, Hyun Woo, Patricia A Shewokis, Scott C Bunce, and Banu Onaral. Cognitive workload and learning assessment during the implementation of a next-generation air traffic control technology using functional near-infrared spectroscopy. IEEE Transactions on Human-Machine Systems, 44(4):429-440, 2014.

[8] Mingming Ma, Shouyun Hu, Longsheng Wang, and Erwin Appel. The distribution process of traffic contamination on roadside surface and the influence of meteorological conditions revealed by magnetic monitoring. Environmental monitoring and assessment, 188(11):650, 2016.

[9] Nishu Singla. Motion detection based on frame difference method. International Journal of Information \& Computation Technology, 4(15):1559-1565, 2014.

[10] Andrews Sobral and Antoine Vacavant. A comprehensive review of background subtraction algorithms evaluated with synthetic and real videos. Computer Vision and Image Understanding, 122:4-21, 2014. 